\title{
Outpatient care is noninferior to inpatient care for low-risk patients with acute pulmonary embolism
}

T he published findings of a randomized, multinational, openlabel trial have shown that outpatient care is noninferior to inpatient care for low-risk patients with pulmonary embolism (PE). "Our findings have the potential to transform the clinical management of PE by shifting management of a substantial proportion of low-risk patients to the outpatient setting," comments Dr Drahomir Aujesky, lead author on the Lancet paper.

"Although practice guidelines recommend outpatient care for clinically stable patients with acute PE," says Aujesky, "most patients with PE continue to be admitted to the hospital." Professor Harry Büller, a prominent expert on venous thromboembolism (VTE) who was not involved in the trial, agrees with Dr Aujesky and elaborates that "whilst out-of-hospital treatment for deep-vein thrombosis (DVT) has taken off like a bush fire and is used for a great majority of patients, there has been a reluctance to send patients with PE home, even though we agree that it is the same disease (VTE)."

According to Dr Aujesky, a "lack of randomized trials comparing the effectiveness and safety of outpatient versus inpatient care for PE" is one of "the main barriers to outpatient management of PE." Aujesky and colleagues, therefore, initiated a noninferiority trial of outpatient versus inpatient care for patients with acute symptomatic PE and a low risk of death.

In total, 344 patients classified as having risk class I or II on the PE Severity Index were enrolled in the trial at 19 emergency departments in Belgium, France, Switzerland, and the USA. Individuals assigned to outpatient care were discharged from hospital no more than $24 \mathrm{~h}$ after randomization, after being taught to self administer subcutaneous injections of $1 \mathrm{mg} / \mathrm{kg}$ enoxaparin twice daily for at least 5 days and until the international normalized ratio was 2.0 or more for 2 consecutive days. Patients assigned to inpatient care were treated with the same regimen of subcutaneous enoxaparin injection and were discharged from hospital when their treating doctor (who was not involved in the study itself) determined that they were ready to go home. All patients were prescribed oral anticoagulation with vitamin $\mathrm{K}$ antagonists for at least 90 days.

The time from presentation at the emergency department to randomization was $13.9 \mathrm{~h}$ and $13.3 \mathrm{~h}$ for the 'outpatients' and 'inpatients', respectively $(P=0.24)$. Mean time in the hospital after randomization was 0.5 days for the individuals assigned to outpatient care and 3.9 days for patients assigned to receive inpatient care.

The primary outcome of the study was recurrent symptomatic PE, or new or recurrent symptomatic DVT, within 90 days of randomization. In the primary analysis of 171 outpatients and 168 inpatients, outpatient care was found to be noninferior to inpatient care for this outcome; no-one in the inpatient group and only one person being treated as an outpatient experienced recurrent VTE within 90 days (at day 83).

Outpatient care was also noninferior for all-cause mortality within 90 days and major bleeding within 14 days in the primary analysis. The noninferiority threshold ( $4 \%$ difference) was slightly exceeded for major bleeds within 90 days of randomization in the primary analysis; however, per-protocol analysis (which excluded eight patients assigned to the outpatient group but who were not treated as outpatients, and 14 patients assigned to the inpatient group but who were not treated as inpatients) showed noninferiority for this secondary outcome.

As Dr Aujesky highlights, the findings "are consistent with previous nonrandomized studies and systematic reviews showing that outpatient care of $\mathrm{PE}$ is associated with low rates of recurrent VTE, major bleeding, and death."

All patients were asked to complete a 5-point Likert scale questionnaire 14 days

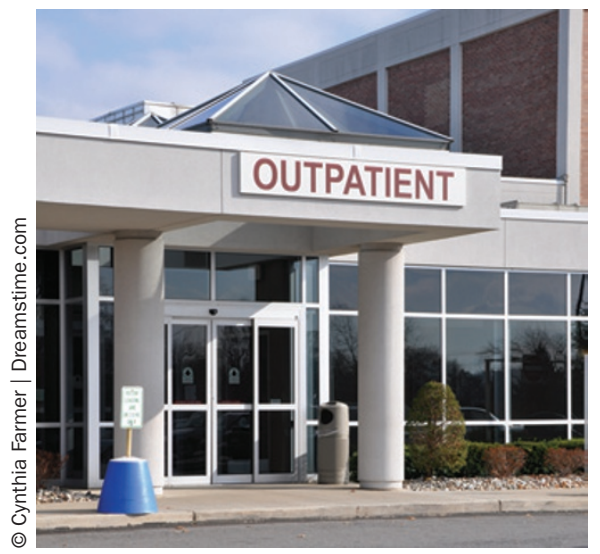

after randomization, to rate their overall satisfaction with care and their treatment preferences. Notably, similar proportions of outpatients and inpatients (92\% and $95 \%$, respectively; $P=0.39$ ) were 'satisfied' or 'very satisfied' with their medical care. Although 14\% of outpatients would have preferred longer treatment in the hospital before discharge, $29 \%$ of the inpatients said that they would have preferred to be treated as an outpatient from the start of their treatment regimen.

The proportion of all patients presenting with PE who were considered to be at 'low risk' and eligible for the trial was approximately $30 \%$. Professor Büller wonders if we can further increase the proportion of patients with $\mathrm{PE}$ who are sent home: "we now need to try to identify whether there is a further subgroup of patients who are not currently classified as 'low risk' but who have a low enough risk for outpatient care ... the future is to identify the group of patients that needs $12 \mathrm{~h}$ or 1 day observation before the decision is made as to whether to send them home."

Bryony M. Mearns 